\title{
MEASUREMENTS OF HYDROGEN SOLUBILITY IN NITROBENZENE/ANILINE MIXTURES
}

\begin{abstract}
Aleksandra Srebniak ${ }^{1}$, Manfred Kraut ${ }^{* 2}$, Oliver Görke ${ }^{2}$, Paweł Sobieszuk ${ }^{* 1}$
${ }^{1}$ Warsaw University of Technology, Faculty of Chemical and Process Engineering, Warynskiego 1, 00-645 Warsaw, Poland

${ }^{2}$ Institute for Micro Process Engineering/Karlsruhe Institute of Technology/Hermann-vonHelmholtz-Platz 1, 76344 Eggenstein-Leopoldshafen, Germany

Measurements of hydrogen solubility in various nitrobenzene-aniline mixtures were conducted in an autoclave reactor with a stirrer and control of temperature. The solubility of hydrogen was measured at 7 different values of temperature $\left(30{ }^{\circ} \mathrm{C}, 40^{\circ} \mathrm{C}, 50^{\circ} \mathrm{C}, 90^{\circ} \mathrm{C}, 130{ }^{\circ} \mathrm{C}, 170{ }^{\circ} \mathrm{C}, 210{ }^{\circ} \mathrm{C}\right.$, respectively), 3 values of stirrer rotation speed (1200 rpm, $1600 \mathrm{rpm}, 2000 \mathrm{rpm}$, respectively) and a range of pressure of $20-30 \mathrm{bar}$. Moreover, pure aniline, pure nitrobenzene and their mixtures with different concentrations were used. In the next step, values of Henry's constant were calculated. Based on experimental data a dependence of Henry's constant on temperature for pure aniline and pure nitrobenzene was proposed. Additionally, for each temperature correlations between Henry's constant and aniline's concentration in mixture of nitrobenzene-aniline were found.
\end{abstract}

Keywords: solubility, Henry's constant, hydrogenation

\section{INTRODUCTION}

The annual world production of aniline amounts to 3.8 million tones and increases from year to year (Kahl et al., 2011). Originally, aniline was produced in the process of reduction of nitrobenzene by cheap reagents, for instance, sulphuric and nitric acid in the presence of an iron catalyst (Morrison and Boyd, 2002). This process generated large quantities of waste reagents and was finally replaced by catalytic hydrogenation of nitrobenzene (Machado, 2007). The amount of hydrogen dissolved in the liquid mixture directly affects and limits this process. Three-phase-hydrogenation processes seem to be a promising way of enhancing the space-time yield (Ramachardan and Chaudhari, 1983; Tatterson, 1991), especially in microreactors such as microfall film reactors (Yeong et al., 2003), therefore, it is of high interest to know the solubility and also the solution rate of hydrogen in nitrobenzene/aniline mixtures. With thermodynamic solubility and kinetic solution rate data, it would be possible to build up a computer-aided model of a nitrobenzene hydrogenation reactor. However, there is a lack of experimental data concerning the solubility of hydrogen in mixtures of nitrobenzene/aniline. Therefore, the aim of this work is to measure hydrogen solubility in nitrobenzene/aniline mixtures under different process conditions (influence of temperature, mixing intensity and liquid phase composition). The experimental data obtained is essential for nitrobenzene hydrogenation with a catalyst in further microreactors' investigation.

*Corresponding authors, e-mail: Pawel.Sobieszuk@pw.edu.pl,manfred.kraut@kit.edu 


\section{EXPERIMENTAL SETUP}

The experiments were carried out in an autoclave reactor with a stirrer. The autoclave was filled with a mixture of nitrobenzene and aniline to half of its volume $(85 \mathrm{ml})$ and sealed up (Fig. 1). Before the first experiment, on each day and after every following one, the reactor was purged with nitrogen for half an hour when the inlet and outlet valves were open and the stirrer was slowly rotating. The experiments were conducted for five mixtures: $100 \%$ nitrobenzene volume content, $75 \%$ nitrobenzene $+25 \%$ aniline, $50 \%$ nitrobenzene $+50 \%$ aniline, $25 \%$ nitrobenzene $+75 \%$ aniline and $100 \%$ aniline, respectively. Each mixture was investigated at 7 temperatures: $30^{\circ} \mathrm{C}, 40^{\circ} \mathrm{C}, 50{ }^{\circ} \mathrm{C}, 90{ }^{\circ} \mathrm{C}, 130^{\circ} \mathrm{C}$, $170{ }^{\circ} \mathrm{C}$ and $210{ }^{\circ} \mathrm{C}$, respectively. For $25 \%$ nitrobenzene $+75 \%$ aniline mixture and $100 \%$ aniline the results for $210{ }^{\circ} \mathrm{C}$ were omitted. This temperature is higher than the boiling point $\left(184{ }^{\circ} \mathrm{C}\right)$ of aniline and under atmospheric pressure at the beginning of the experiments these liquids were boiling, which led to incorrect results. To perform the experiments, the inlet and outlet gas nozzles were tightly closed and hydrogen was dosed with the mass flow controller into the autoclave reactor. Gas pressure in the pipe before the inlet valve was recorded. When the pipe filled up with sufficient amount of hydrogen, its flow was switched off. Next the inlet valve to the autoclave was opened. The moment the pressure inside the autoclave had the desired value, the inlet valve was quickly closed and the stirrer was set on the desired speed of rotation. The first value of pressure (for zero time) was written down. For each temperature the experiments were carried out at three stirrer rotation speeds: $1200 \mathrm{rpm}, 1600 \mathrm{rpm}$ and $2000 \mathrm{rpm}$, respectively (for pure nitrobenzene only under 1200 and $1600 \mathrm{rpm}$ ). Finally, each experiment was replicated three times for each stirrer rotation speed, filling the reactor with such an amount of hydrogen that the initial pressure at time zero was in the range of $20-30$ bar. The pressure of gas in reactor was measured. Hydrogen absorption by the liquid was accompanied by a pressure decrease until a steady-state has been reached. Based on the final value of gas pressure, the solubility of hydrogen in the mixture was determined.

a)

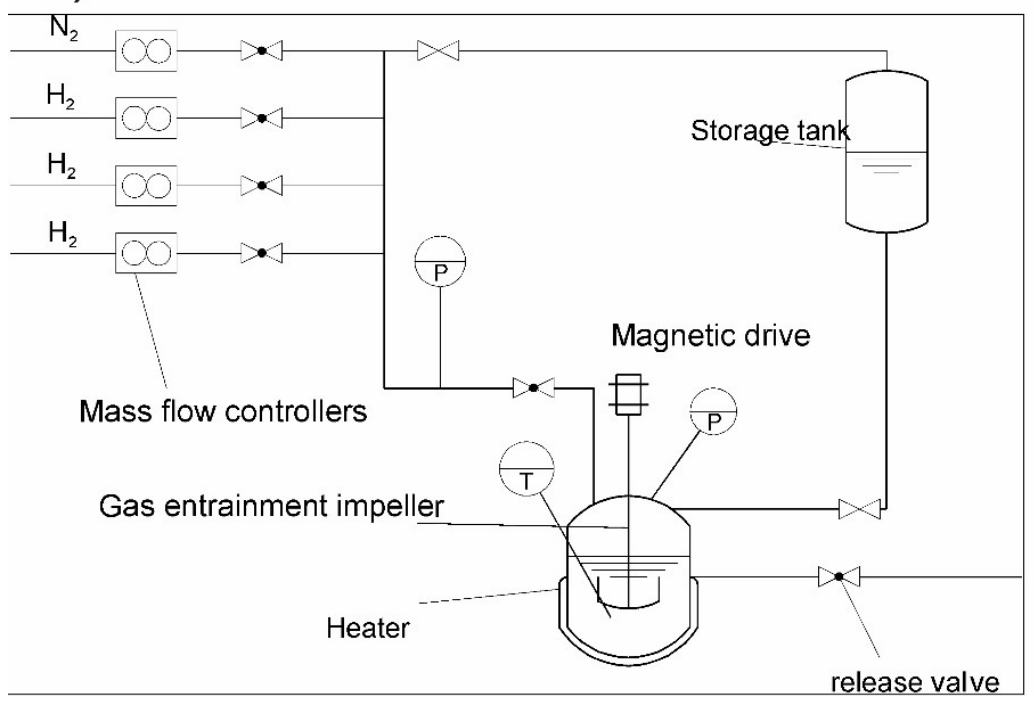

b)

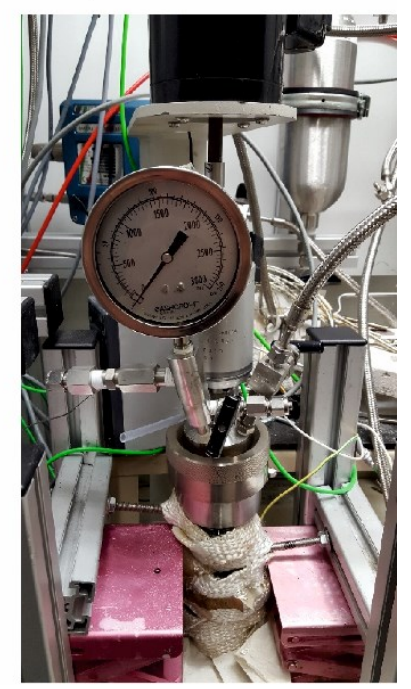

Fig. 1. Experimental set-up: a) flow scheme , b) image of stirred autoclave

\section{METHODOLOGY}

Pressure drop, $p$, observed in the course of experiments (a difference between the initial pressure and that at the moment of its stabilization) is a sum of hydrogen pressure $p_{H 2}$ and the vapour pressure of liquid $p_{L}$. The values of $p_{L}$ were calculated according to the Antoine's equation for pure nitrobenzene and pure aniline. In the case of nitrobenzene/aniline mixtures, vapour pressure was calculated from the 
Raoult law. In this way, the pressure drop observed as a result of hydrogen absorption $\Delta p$ was found and used to count the number of moles of absorbed hydrogen. Under the experimental conditions the compressibility factor equals 1 and for the number of moles of absorbed gas the ideal gas law is applicable:

$$
n=\frac{\Delta p V_{G}}{T R}
$$

The amount of absorbed hydrogen per liquid volume $L\left[\mathrm{~mol} / \mathrm{m}^{3}\right]$ was found from the equation:

$$
L=\frac{n}{V_{L}}
$$

In the next step the Henry' constant $H\left[\mathrm{bar}^{3} / \mathrm{mol}\right]$ was determined as:

$$
H=\frac{p_{H 2}}{L}
$$

In Eq. (3) hydrogen pressure $p_{H 2}$ is exactly what has been measured at each measurement point. For aniline Henry's constant was described as $H_{A N}$, for pure nitrobenzene as $H_{N B}$ and for mixture as $H_{M}$, respectively. Values $L$ are dependent on pressure, while Henry's constant $H$ calculated from Eq. (3) is independent of pressure within the range of pressure used.

\section{RESULTS AND DISCCUSION}

In all experiments the changes of pressure in time were measured. As an example, the pressure drops are shown in Fig. 2 at $1200 \mathrm{rpm}, 1600 \mathrm{rpm}$ and $2000 \mathrm{rpm}$, respectively for 50\% nitrobenzene $+50 \%$ aniline mixture at $130{ }^{\circ} \mathrm{C}$. The pressure at the start of the experiment was similar for this mixture at all three stirrer rotation speeds. It can be deduced that the duration time of experiment was shorter for higher stirrer rotation speeds and in all cases the steady-states conditions were achieved.

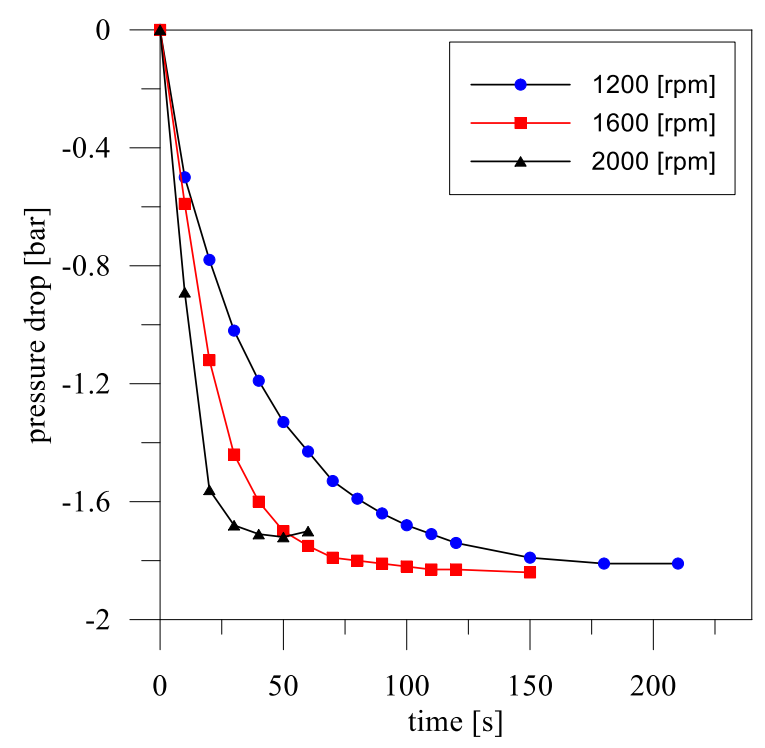

Fig. 2. Pressure drop for $50 \%$ volumetric concentration of aniline at $130^{\circ} \mathrm{C}$

Based on the steady-state conditions in all experiments, the values of $\Delta p$ were determined. Using Eq. (2) the amount of absorbed hydrogen was calculated and presented in Fig. 3. As can be seen in Fig. 3 , an increase in the amount of hydrogen in nitrobenzene/aniline mixtures was observed with the increasing temperature. Similar results for hydrogen were found for other organic substances. Brunner 
(1985) investigated solubility of hydrogen in $n$-hexane, $n$-octane, toluene, acetonitrile, 1,4-dioxane, $\mathrm{N}, \mathrm{N}$-dimethylformamide, $n$-decane, tetrahydrofuran, 1- methylpyrrolidone-2, acetone at $298.15 \mathrm{~K}$, $323.15 \mathrm{~K}$ and $373.15 \mathrm{~K}$, respectively, and partially at pressures up to $14 \mathrm{MPa}$. He found that the solubility of hydrogen increases with the increasing temperature.
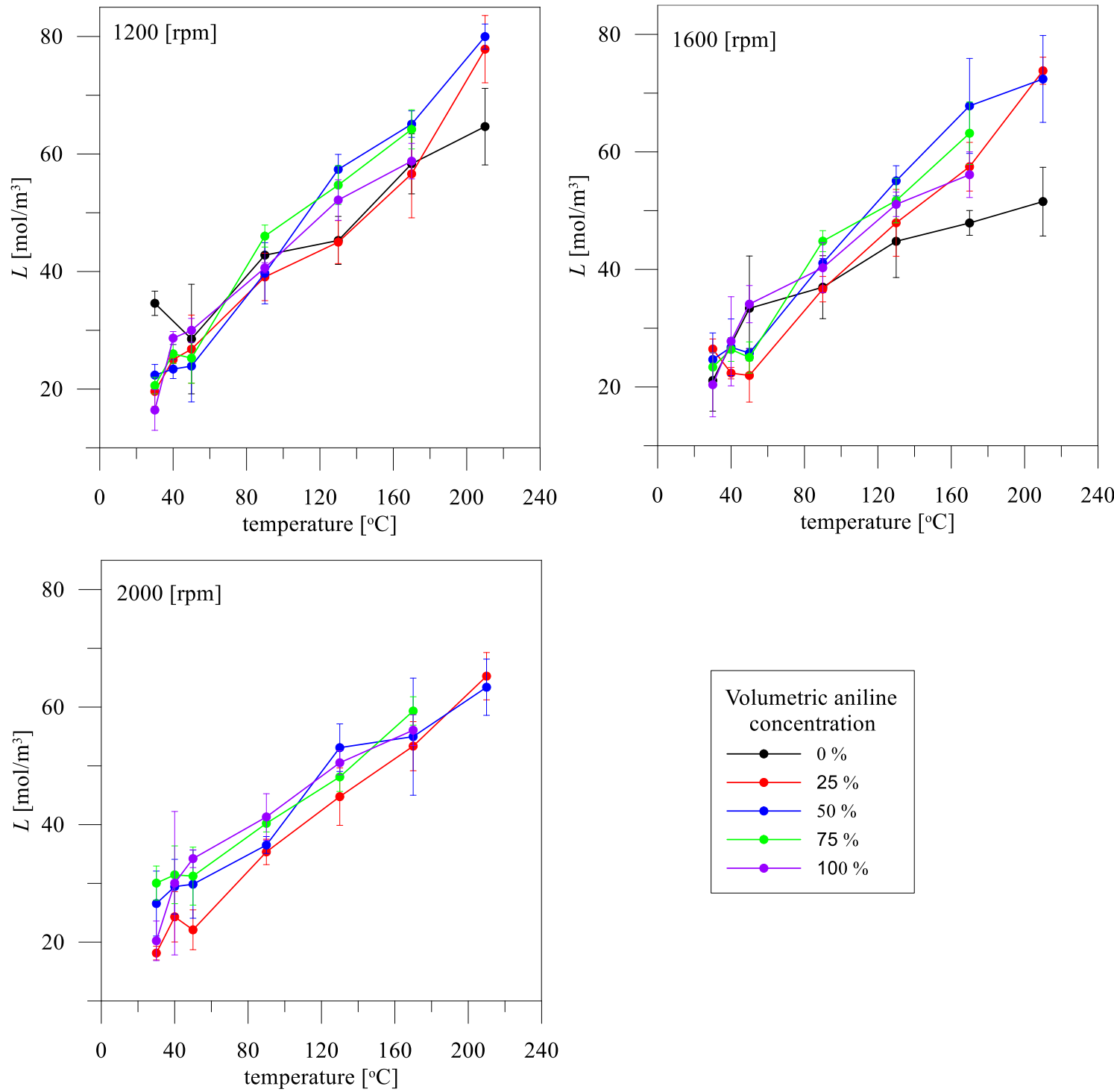

Fig. 3. Amount of absorbed hydrogen per liquid volume in nitrobenzene/aniline mixtures at 1200,1600 and $2000 \mathrm{rpm}$

D'Angelo and Francesconi (2001) investigated solubility of hydrogen in liquid alcohols: methanol, ethanol, 1-propanol and 1-butanol within the range of temperatures between $298.15 \mathrm{~K}$ and $525.15 \mathrm{~K}$ and at pressures between 3.6 MPa and 10 MPa. They concluded that the solubility of hydrogen in all studied alcohols increased with increasing temperature and pressure. An exemplary influence of the stirrer rotation speed on solubility of hydrogen is shown in Figs. $4-5 . L$ is practically independent of the stirrer rotation speed, when error bars of experimental data are in the limit of experimental scatters.

In order to present results as pressure, independent $H$ values are calculated. For pure liquids, the Henry's constants were calculated based on the results of experiments and Eq. (3). The average values of these constants were calculated for the experiment temperatures and for three stirrer rotation speeds. The $H$ values are presented in Fig. 6. 


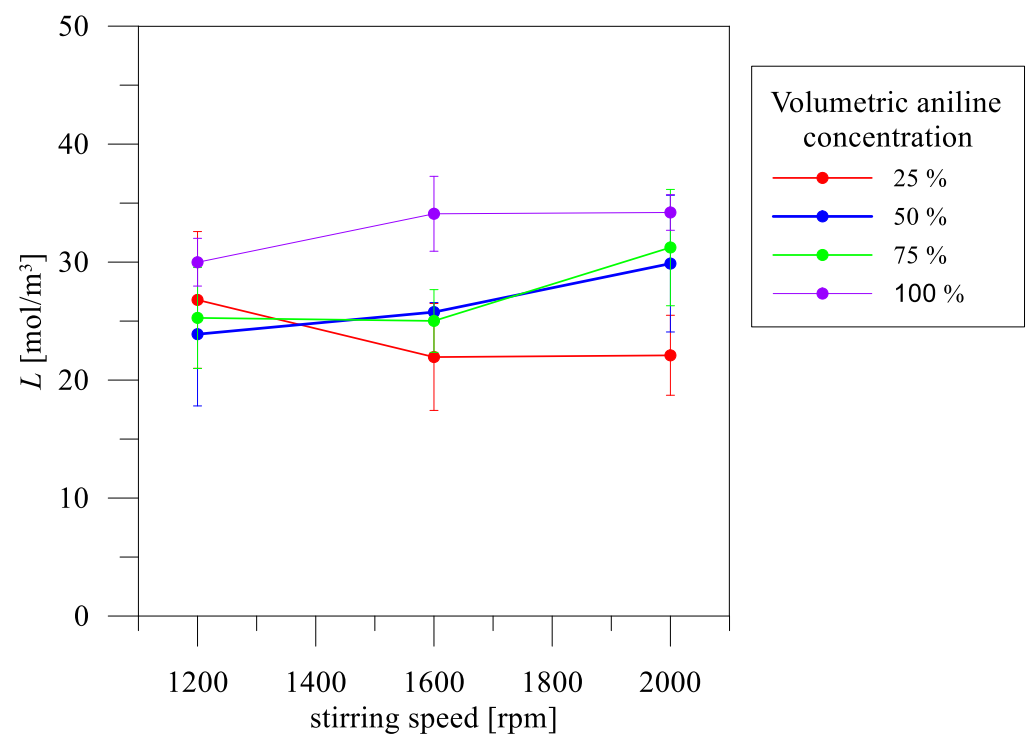

Fig. 4. Amount of absorbed hydrogen per liquid volume in nitrobenzene/aniline mixtures at $50{ }^{\circ} \mathrm{C}$

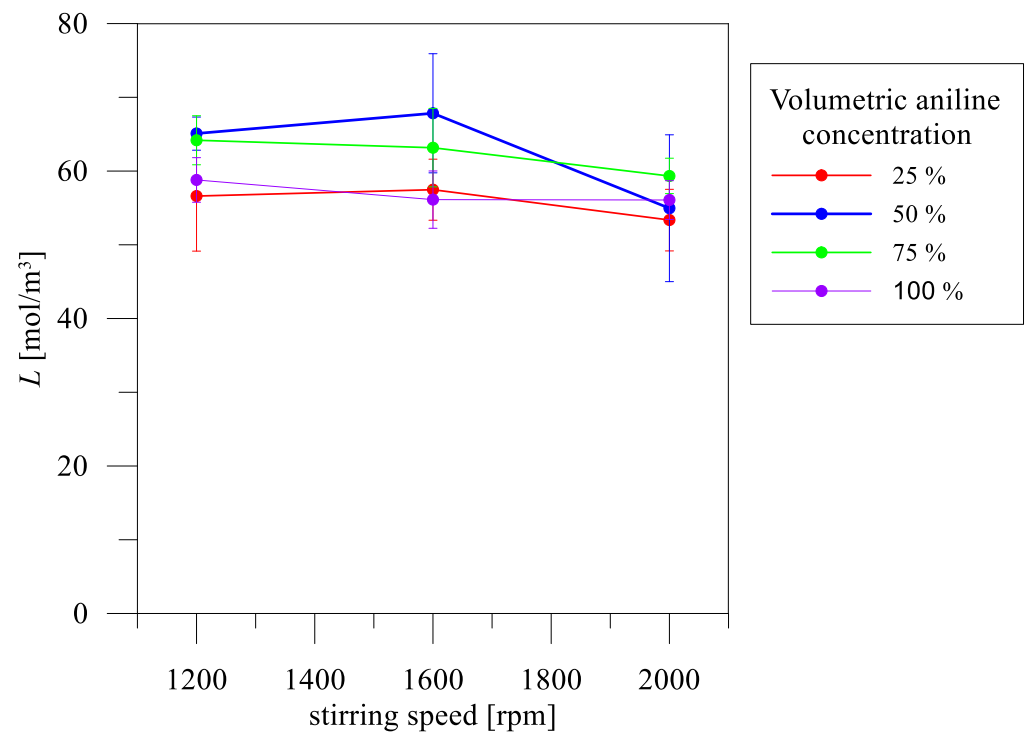

Fig. 5. Amount of absorbed hydrogen per liquid volume in nitrobenzene/aniline mixtures at $170{ }^{\circ} \mathrm{C}$

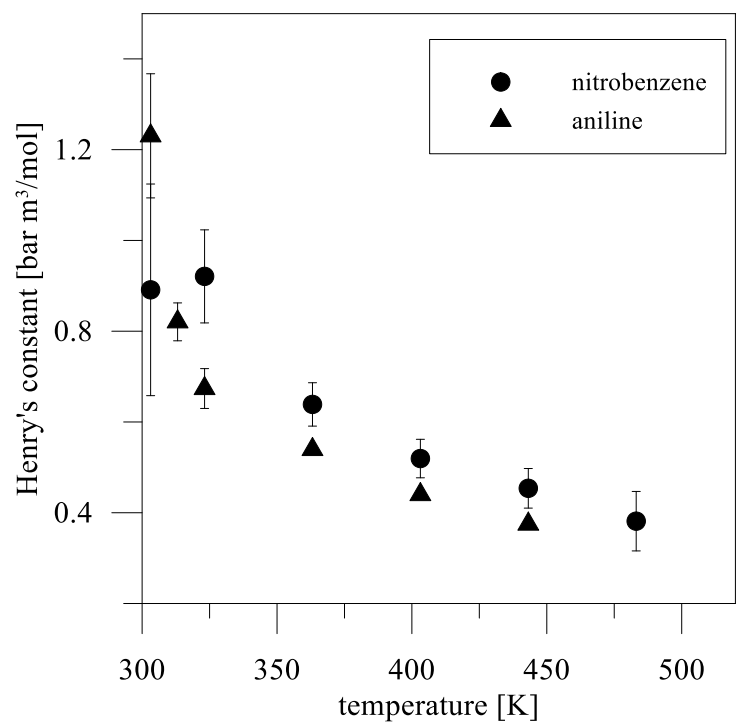

Fig. 6. Henry’s constants for pure nitrobenzene and aniline 
In the next step, the dependences of Henry's constants in function of temperature for pure nitrobenzene $\left(H_{N B}\right)$ and pure aniline $\left(H_{A N}\right)$ were determined:

$$
\begin{gathered}
\log H_{N B}=1.2268-0.0053 \cdot T+4.06 \cdot 10^{-6} \cdot T^{2} \\
\log H_{A N}=2.4105-0.0108 \cdot T+10^{-5} \cdot T^{2}
\end{gathered}
$$

The form of correlations (4) and (5) is widely cited based on the publications of Barret (1966) and Danckwerts (1970), who investigated the influence of temperature on gas solubility in liquids.

To compare the experimental values of Henry's constants with those calculated from Eqs. $(4,5)$, parity plots were presented in Fig. 7 for nitrobenzene and in Fig. 8 for aniline, respectively. As can be seen Eq. (4) is accurate with an error of $\pm 10 \%$, while Eq. (5) with an error of $\pm 16 \%$.

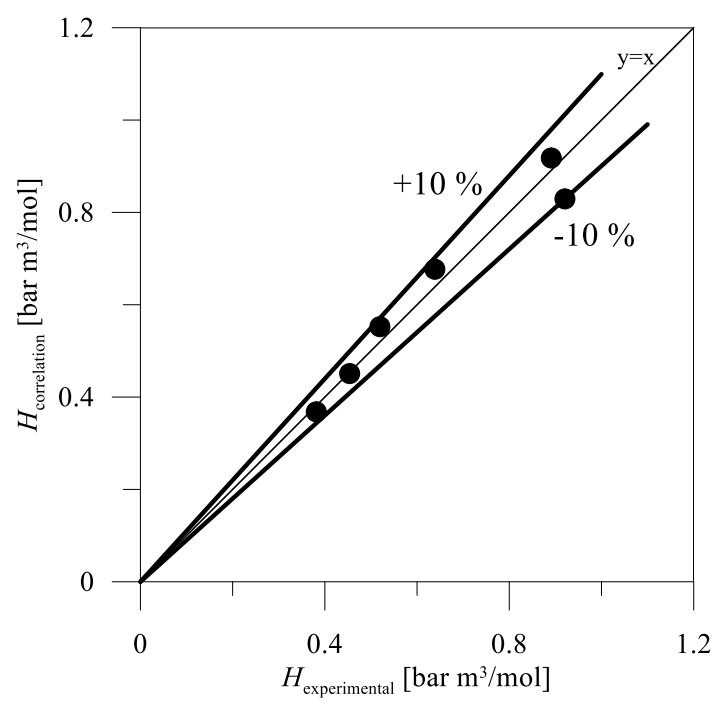

Fig. 7. Parity plot of Eq. (4)

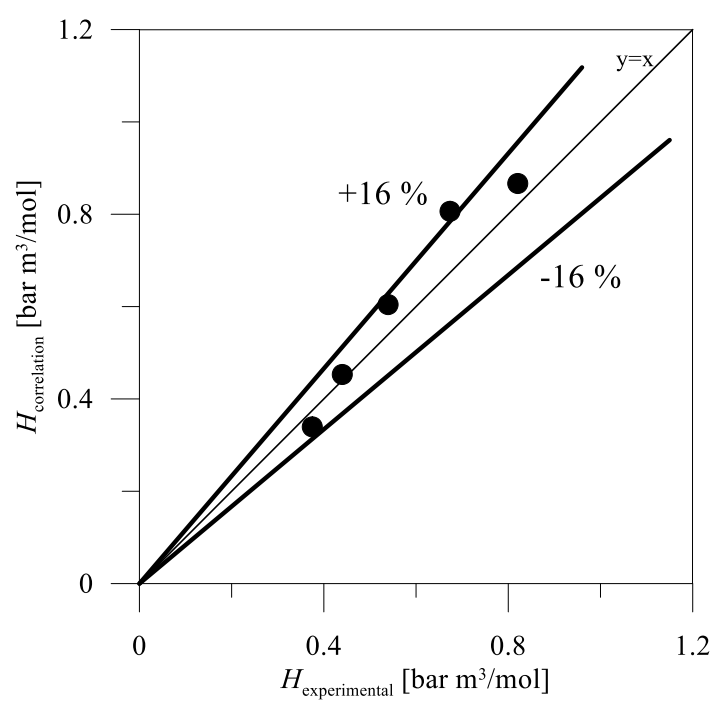

Fig. 8. Parity plot of Eq. (5)

Finally, for temperature in the range of $40{ }^{\circ} \mathrm{C}-170{ }^{\circ} \mathrm{C}$, the values of the Henry's constant for nitrobenzene/aniline mixtures are presented in Fig. 9. As can be seen, $H_{M}$ slightly decreases with increase of aniline concentration, which is in agreement with data in Fig. 6. 


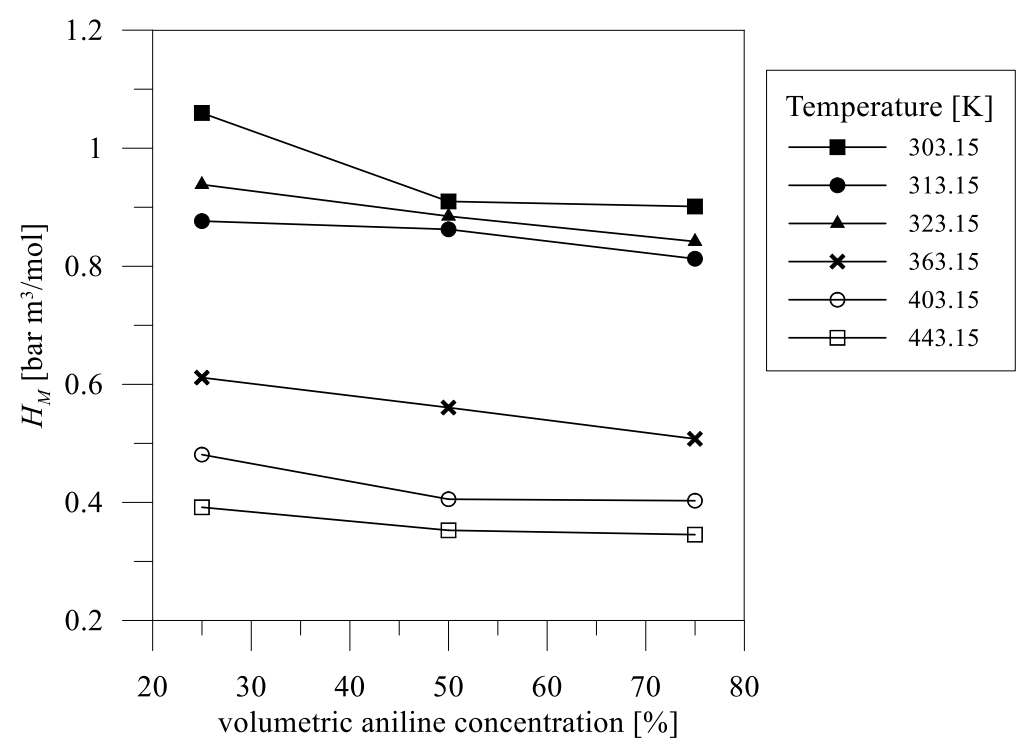

Fig. 9. Henry's constant values for aniline/nitrobenzene mixtures

\section{CONCLUSIONS}

The measurements of hydrogen absorption in nitrobenzene/aniline mixtures were carried out in an autoclave reactor equipped with a stirrer for a wide range of temperature, concentration of aniline and stirrer rotation speed. It was found that the amount of absorbed hydrogen in these mixtures decreases with decreasing temperature in the range of $30{ }^{\circ} \mathrm{C}-210{ }^{\circ} \mathrm{C}$, and under pressure between 20 and 30 bar. Similar effect for other organic liquids is reported in literature. Based on the results of experiments, the Henry's constants as a function of temperature for pure nitrobenzene and aniline were experimentally determined and adequate correlations were proposed. Finally, values of the Henry's constant for nitrobenzene/aniline mixtures at different temperatures were presented.

The data determined represent an important extension of thermodynamic solubility for such a basic reaction mixture system of nitrobenzene, aniline and hydrogen. As the next step, it should further be extended by including water as reaction product in the system. The data obtained can be implemented to establish a better computational model of a three-phase hydrogenation reactor.

\section{SYMBOLS}

$H \quad$ Henry's constant, bar $\cdot \mathrm{m}^{3} / \mathrm{mol}$

$L \quad$ amount of hydrogen absorbed per liquid volume, $\mathrm{mol} / \mathrm{m}^{3}$

$n \quad$ number of mole of absorbed hydrogen

$p \quad$ pressure, $\mathrm{Pa}$

$R \quad$ gas constant, $\mathrm{J} /(\mathrm{mol} \cdot \mathrm{K})$

$T \quad$ temperature, $\mathrm{K}$

$V \quad$ volume, $\mathrm{m}^{3}$

$\Delta p \quad$ pressure drop, $\mathrm{Pa}$

\section{Subscripts}

AN aniline

$G \quad$ gas

H2 hydrogen

$N B \quad$ nitrobenzene 


$\begin{array}{ll}L & \text { liquid } \\ M & \text { mixture }\end{array}$

\section{REFERENCES}

Barret P.V.L., 1966. Gas absorption on a sieve plate. Ph.D. Thesis, University of Cambridge.

Brunner E., 1985. Solubility of hydrogen in 10 organic solvents at 298.15, 323.15, and 372.15 K. J. Chem. Eng. Data, 30, 269-273. DOI: 10.1021/je00041a010.

Danckwerts P.V., 1970. Gas-Liquid Reaction. Mc Graw-Hill, New York.

d'Angelo J.V.H., Francesconi A.Z., 2001. Gas-liquid solubility of hydrogen in $n$-alcohols $(1 \leq n \leq 4)$ at pressures from 3.6 MPa to $10 \mathrm{MPa}$ and temperatures from $298.15 \mathrm{~K}$ to $525.15 \mathrm{~K}$. J. Chem. Eng. Data, 46, 671-674. DOI: $10.1021 / \mathrm{je} 000189 \mathrm{u}$.

Kahl T., Schroeder K.W., Lawrence F.R., Marshall W.J., Hoeke H., Jackh R., 2011. Ullmann's encyclopedia of industrial chemistry. Wiley-VCH.

Machado R., 2007. Fundamentals of mass transfer and kinetics for the hydrogenation of nitrobenzene to aniline. Air Products and Chemicals, 1, 1-14.

Morisson R.T., Boyd R.N., 1992. Organic chemistry. Prentice Hall, New Jersey.

Ramachandran P.A., Chaudhari R.V., 1983. Three-phase catalytic reactors. Topics in Chemical Engineering, Vol. 2, Chapters 9, Gordon and Breach Science Publishers, Philadelphia.

Tatterson G.B., 1991. Fluid mixing and gas dispersion in agitated tanks. McGraw-Hill, New York.

Yeong K.K., Gavrilidis A., Zapf R., Hessel V., 2003. Catalyst preparation and deactivation issues for nitrobenzene hydrogenation in a microstructured falling film reactor. Catalysis Today, 81, 641-651. DOI: $10.1016 / \mathrm{S} 0920-5861(03) 00162-7$. 